\title{
Hyperbolicity of the Complements of Plane Algebraic Curves
}

\author{
G.Dethloff, G.Schumacher, P.M.Wong
}

\section{Contents}

1 Introduction 1

2 Some tools from Value Distribution Theory 3

3 Some consequences of Brody's techniques 7

4 Nonexistence of algebraic entire curves in generic complements

5 Hyperbolicity of generic complements of at least four curves 10

6 Line systems through intersection points of three quadrics $\overline{12}$

(7) Hyperbolicity of generic complements of three quadrics 16

8 Complements of two quadrics and a line 20

\section{Introduction}

Hyperbolic manifolds have been studied in complex analysis as the generalizations of hyperbolic Riemann surfaces to higher dimensions. Moreover, the theory of hyperbolic manifolds is closely related to other areas (cf. eg. [11]).

However, only very few quasi-projective (non closed) hyperbolic manifolds are known. But one still believes that e.g. the complements of 'most' hypersurfaces in $\mathbb{P}_{n}$ are hyperbolic, if only their degree is at least $2 \mathrm{n}+1$, more precisely:

Conjecture 1.1 Let $\mathcal{C}\left(d_{1}, \ldots, d_{k}\right)$ be the space of $k$ tupels of hypersurfaces $\Gamma=\left(\Gamma_{1}, \ldots, \Gamma_{k}\right)$ in $\mathbb{P}_{n}$, where $\operatorname{deg}\left(\Gamma_{i}\right)=d_{i}$. Then for all $\left(d_{1}, \ldots, d_{k}\right)$ with 
$\sum_{i=1}^{k} d_{i}=: d \geq 2 n+1$ the set $\mathcal{H}\left(d_{1}, \ldots, d_{k}\right)=\left\{\Gamma \in \mathcal{C}\left(d_{1}, \ldots, d_{k}\right): \mathbb{P}_{n} \backslash \bigcup_{i=1}^{k} \Gamma_{i}\right.$ is complete hyperbolic and hyperbolically embedded $\}$ contains the complement of a proper algebraic subset of $\mathcal{C}\left(d_{1}, \ldots, d_{k}\right)$.

For complements of hypersurfaces in $\mathbb{P}_{n}$ this was posed by Kobayashi as 'Problem 3' in his book [10], and later by Zaidenberg in his paper [16].

In this paper, we shall deal with the complements of plane curves i.e. the case $\mathrm{n}=2$.

Other than in the case of 5 lines $(\mathcal{C}(1,1,1,1,1))$, the conjecture was previously proved by $\mathrm{M}$. Green in $[8]$ in the case of a curve $\Gamma$ consisting of one quadric and three lines $(\mathcal{C}(2,1,1,1))$. Furthermore, it was shown for $\mathcal{C}\left(d_{1}, \ldots, d_{k}\right)$, whenever $k \geq 5$, by Babets in [3]. A closely related result by Green in [7] is that for any four non-redundant hypersurfaces $\Gamma_{j}, j=1, \ldots 4$ in $\mathbb{P}_{2}$ any entire curve $f: \mathbb{C} \rightarrow \mathbb{P}_{2} \backslash \bigcup_{j=1}^{4} \Gamma_{j}$ is algebraically degenerate. (The degeneracy locus of the Kobayashi pseudometric was studied by Adachi and Suzuki in [䜣, [2]).

In fact, for generic configurations, any such algebraically degenerate map is constant, hence the conjecture is true for any family $\mathcal{C}\left(d_{1}, \ldots\right.$, $d_{k}$ ) with $k \geq 4$ (cf. cf. Theorem 5.1). This includes the case of a curve $\Gamma$ consisting of 2 quadrics and 2 lines. We also give another proof of Green's result, which yields a slightly stronger result related to the statement of a second main theorem of value distribution theory in this situation.

It seems that the conjecture is the more difficult the smaller $\mathrm{k}$ is. Already the case $\mathrm{k}=3$ seems to be very hard: In $1989 \mathrm{H}$. Grauert worked on the case of a curve $\Gamma$ consisting of 3 quadrics, i.e. $\mathcal{C}(2,2,2)$, in [5], using sophisticated differential geometric methods including Jet-metrics. We believe that the methods developed there might be suited for proving major parts of the conjecture. For the time being, however, certain technical problems still exist with these methods including the case $\mathcal{C}(2,2,2)$.

The main result of this paper (Theorem 17.1) is a proof of the conjecture for 3 quadrics. Our methods are completely different from those used in [5] - instead of differential geometry we use value distribution theory: For any pair of quadrics which intersect transversally, there are 6 lines through the intersection points, out of which 4 are in general position. We first show that we can assign a set of 12 lines in general position to any generic system $C$ of 3 quadrics. Let $f: \mathbb{C} \rightarrow \mathbb{P}_{2} \backslash C$ be an entire holomorphic curve. Our method now essentially consists of showing that the defect of $f$ with respect to the above 12 lines had to be at least equal to 4 unless $f$ is algebraically degenerate. (For 
technical reasons our exposition is based on the Second Main Theorem rather than the defect relation). The last step is to show that this fact is actually sufficient for generic complements of 3 quadrics to be complete hyperbolic and hyperbolically embedded.

For $\mathcal{C}(2,2,1)$, i.e. two quadrics and a line, our result states the existence of an open set, which contains a quasi-projective set of codimension one, of configurations, where the conjecture is true (Theorem 8.11). The somewhat lengthy proof is based on a generalized Borel lemma. With the same methods we prove that also the complement of three generic Fermat quadrics is hyperbolic.

The paper is organized as follows: In section 2 we collect, for the convenience of the reader, some basics from value distribution theory, and, in section 3, some consequences from Brody's techniques for later reference. In section 4 we prove some 'algebraic' hyperbolicity of generic complements of certain curves. Next, in section 5 we prove Theorem 5.1. In section 6 we study linear systems of lines associated to systems of 3 quadrics. Section 7 contains the proof of Theorem 7.1. In section 8 we treat complements of two quadrics and a line and complements of three Fermat quadrics.

The first named author would like to thank S. Frankel (Nantes), H. Grauert (Göttingen), S. Kosarew (Grenoble) and M. Zaidenberg (Grenoble) for valuable discussions, the Department of Mathematics at Notre Dame for its hospitality, and the DFG, especially the 'Schwerpunkt Komplexe Mannigfaltigkei-

ten' in Bochum for support. The second named author would like to thank H. Grauert, W.Stoll (Notre Dame) and M.Zaidenberg for valuable discussions, and the Department of Mathematics at Notre Dame and the SFB 170 in Göttingen for its hospitality and the Schwerpunkt 'Komplexe Mannigfaltigkeiten' for support. The third named author would like to thank the SFB 170 and the NSF for partial support.

\section{Some tools from Value Distribution The- ory}

In this section we fix some notations and quote some facts from Value Distribution Theory. We give references but do not trace these facts back to the original papers.

We define the characteristic function and the counting function, and give 
some formulas for these.

Let $\|z\|^{2}=\sum_{j=0}^{n}\left|z_{j}\right|^{2}$, where $\left(z_{0}, \ldots, z_{n}\right) \in \mathbb{C}^{n+1}$, let $\Delta_{t}=\{\xi \in \mathbb{C}:|\xi|<$ $t\}$, and let $d^{c}=(i / 4 \pi)(\bar{\partial}-\partial)$. Let $r_{0}$ be a fixed positive number and let $r \geq r_{0}$. Let $f: \mathbb{C} \rightarrow \mathbb{P}_{n}$ be entire, i.e. $f$ can be written as $f=\left[f_{0}: \ldots: f_{n}\right]$ with holomorphic functions $f_{j}: \mathbb{C} \rightarrow \mathbb{C}, j=0, \ldots, n$ without common zeroes. Then the characteristic function $T(f, r)$ is defined as

$$
T(f, r)=\int_{r_{0}}^{r} \frac{d t}{t} \int_{\Delta_{t}} d d^{c} \log \|f\|^{2}
$$

Let furthermore $D=\{P=0\}$ be a divisor in $\mathbb{P}_{n}$, given by a homogeneous polynomial $P$. Assume $f(\mathbb{C}) \not \subset \operatorname{support}(D)$. Let $n_{f}(D, t)$ denote the number of zeroes of $P \circ f$ inside $\Delta_{t}$ (counted with multiplicities). Then we define the counting function as

$$
N_{f}(D, r)=\int_{r_{0}}^{r} n_{f}(D, t) \frac{d t}{t}
$$

Stokes Theorem and transformation to polar coordinates imply (cf. [15]):

$$
T(f, r)=\frac{1}{4 \pi} \int_{0}^{2 \pi} \log \|f\|^{2}\left(r e^{i \vartheta}\right) d \vartheta+O(1) .
$$

The characteristic function as defined by Nevanlinna for a holomorphic function $f: \mathbb{C} \rightarrow \mathbb{C}$ is

$$
T_{0}(f, r)=\frac{1}{2 \pi} \int_{0}^{2 \pi} \log ^{+}\left|f\left(r e^{i \vartheta}\right)\right| d \vartheta .
$$

For the associated map $[f: 1]: \mathbb{C} \rightarrow \mathbb{P}_{1}$ one has

$$
T_{0}(f, r)=T([1: f], r)+O(1)
$$

(cf. [9]).

By abuse of notation we will, from now on, for a function $f: \mathbb{C} \rightarrow \mathbb{C}$, write $T(f, r)$ instead of $T_{0}(f, r)$. Furthermore we sometimes use $N(f, r)$ instead of $N_{f}\left(\left[z_{0}=0\right], r\right)$.

The concept of finite order is essential for later applications.

Definition 2.1 Let $s(r)$ be a positive, monotonically increasing function defined for $r \geq r_{0}$. If

$$
\varlimsup_{r \rightarrow \infty} \frac{\log s(r)}{\log r}=\lambda
$$

then $s(r)$ is said to be of order $\lambda$. For entire $f: \mathbb{C} \rightarrow \mathbb{P}_{n}$ or $f: \mathbb{C} \rightarrow \mathbb{C}$ we say that $f$ is of order $\lambda$, if $T(f, r)$ is. 
Remark 2.2 Let $f=\left[f_{0}: \ldots: f_{n}\right]: \mathbb{C} \rightarrow \mathbb{P}_{n}$ be a holomorphic map of finite order $\lambda$. Then $\log T(f, r)=O(\log r)$.

We need the following:

Lemma 2.3 Assume that $f: \mathbb{C} \rightarrow \mathbb{P}_{n}$ is an entire map and misses the divisors $\left\{z_{j}=0\right\}$ for $j=0, \ldots, n$ (i.e. the coordinate hyperplanes of $\mathbb{P}_{n}$ ). Assume that $f$ has order at most $\lambda$. Then $f$ can be written as $f=\left[1: f_{1}: \ldots: f_{n}\right]$ with $f_{j}(\xi)=e^{P_{j}(\xi)}$, where the $P_{j}(\xi)$ are polynomials in $\xi$ of degree $d_{j} \leq \lambda$.

Proof: We write $f=\left[1: f_{1}: \ldots: f_{n}\right]$ with holomorphic $f_{j}: \mathbb{C} \rightarrow \mathbb{C} \backslash\{0\}$. Now we get with equations (11) and (2) for $j=1, \ldots, n$ :

$$
T\left(f_{j}, r\right)=T\left(\left[1: f_{j}\right], r\right)+O(1) \leq T(f, r)+O(1),
$$

hence the $f_{j}$ are nonvanishing holomorphic functions of order at most $\lambda$. This means that

$$
\lim \sup _{r \rightarrow \infty} \frac{T\left(f_{j}, r\right)}{r^{\lambda+\epsilon}}=0
$$

for any $\epsilon>0$. From this equation our assertion follows with the Weierstraß theorem as it is stated in [9].

The previous Lemma is helpful because we can use it to 'calculate' $T(f, r)$ by the Ahlfors-Lemma (cf. [14])

Lemma 2.4 Let $P_{0}, \ldots, P_{n}$ be polynomials of degree at most $\lambda \in \mathbb{N}$. Let $\alpha_{j} \in$ $\mathbb{C}$ be the coefficients of $x^{\lambda}$ in $P_{j}$ (possibly equal to zero). Let $L\left(\alpha_{0}, \ldots, \alpha_{n}\right)$ be the length of the polygon defined by the convex hull of the $\alpha_{0}, \ldots, \alpha_{n}$. If

$$
f=\left[e^{P_{0}}: \ldots: e^{P_{n}}\right]: \mathbb{C} \rightarrow \mathbb{P}_{n}
$$

then

$$
\lim _{r \rightarrow \infty} \frac{T(f, r)}{r^{\lambda}}=\frac{L\left(\alpha_{0}, \ldots, \alpha_{n}\right)}{2 \pi}
$$

We state the First and the Second Main Theorem of Value Distribution Theory which relate the characteristic function and the counting function (cf. [13]):

Let $f: \mathbb{C} \rightarrow \mathbb{P}_{n}$ be entire, and let $D$ be a divisor in $\mathbb{P}_{n}$ of degree $d$, such that $f(\mathbb{C}) \not \subset \operatorname{support}(D)$. Then: 


\section{First Main Theorem}

$$
N_{f}(D, r) \leq d \cdot T(f, r)+O(1)
$$

Another way of stating this theorem is the following: The quantity

$$
\delta_{f}(D)=\liminf _{r \rightarrow \infty}\left(1-\frac{N_{f}(D, r)}{d \cdot T(f, r)}\right)
$$

is called defect of $D$ with respect to $f$. Then

$$
\delta_{f}(D) \geq 0 \text {. }
$$

Assume now that $f(\mathbb{C})$ is not contained in any hyperplane in $\mathbb{P}_{n}$, and let $H_{1}, \ldots, H_{q}$ be distinct hyperplanes in general position. Then

\section{Second Main Theorem}

$$
(q-n-1) T(f, r) \leq \sum_{j=0}^{q} N_{f}\left(H_{j}, r\right)+S(r)
$$

where $S(r) \leq O(\log (r T(f, r)))$ for all $r \geq r_{0}$ except for a set of finite Lebesque measure. If $f$ is of finite order, then $S(r) \leq O(\log r)$ for all $r \geq r_{0}$.

We examine how the characteristic function behaves under morphisms of the projective space:

\section{Lemma 2.5 Let}

$$
R=\left[R_{0}: \ldots: R_{N}\right]: \mathbb{P}_{n} \rightarrow \mathbb{P}_{N}
$$

be a morphism with components of degree $p$, and let $f: \mathbb{C} \rightarrow \mathbb{P}_{n}$ be entire. Then

$$
T(R \circ f, r)=p \cdot T(f, r)+O(1)
$$

Proof: Define

$$
\mu\left(\left[z_{0}: \ldots: z_{n}\right]\right)=\frac{\left|R_{0}\right|^{2}+\ldots+\left|R_{N}\right|^{2}}{\left(\left|z_{0}\right|^{2}+\ldots+\left|z_{n}\right|^{2}\right)^{p}}
$$

Since $R$ is a morphism the $R_{j}, j=0, \ldots, N$ have no common zeroes, hence there exist constants $A, B>0$ with $0<A \leq \mu \leq B$ on $\mathbb{P}_{n}$. From that and equation (11) we get:

$$
\begin{aligned}
T(R \circ f, r)-p \cdot T(f, r) & =\frac{1}{4 \pi} \int_{0}^{2 \pi}\left(\log \|R \circ f\|^{2}\left(r e^{i \vartheta}\right)-p \cdot \log \|f\|^{2}\left(r e^{i \vartheta}\right)\right) d \vartheta+O(1) \\
& =\frac{1}{4 \pi} \int_{0}^{2 \pi} \log (\mu \circ f)\left(r e^{i \vartheta}\right) d \vartheta+O(1)
\end{aligned}
$$

In the last term the integral is bounded by $\frac{1}{2} \log A$ and $\frac{1}{2} \log B$ independently of $r$. 


\section{Some consequences of Brody's techniques}

In this section we list briefly some consequences of Brody's techniques for later application. The first is a corollary of a well known theorem of M. Green. It shows how to use entire curves $f: \mathbb{C} \rightarrow \mathbb{P}_{2}$ of finite order to prove hyperbolicity of quasiprojective varieties. The second follows from of a theorem of M.Zaidenberg.

a) The main theorem of [8] implies:

Corollary 3.1 Let $D$ be a union of curves $D_{1}, \ldots, D_{m}$ in $\mathbb{P}_{2}$ such that for all $i=1, \ldots, m$ the number of intersection points of $D_{i}$ with $\bigcup_{j=1, \ldots, m ; j \neq i} D_{j}$ is at least three. Then $\mathbb{P}_{2} \backslash D$ is complete hyperbolic and hyperbolically embedded, if there does not exist a non-constant entire curve $f: \mathbb{C} \rightarrow \mathbb{P}_{2}$ of order at most two which misses $D$.

b) The following proposition shows that the property of a union of curves having hyperbolic complement is essentially a (classically) open condition.

Proposition 3.2 Let $H_{1}, \ldots, H_{m}$ be hypersurfaces in $\mathbb{P}_{2} \times\left(\Delta_{t}\right)^{n}$ for some $t>0, n \in \mathbb{N}$. Let $\pi: \mathbb{P}_{2} \times\left(\Delta_{t}\right)^{n} \rightarrow\left(\Delta_{t}\right)^{n}$ be the projection. Assume that

1) for all $z \in\left(\Delta_{t}\right)^{n}$ and all $i=1, \ldots, m$ the fibers $\pi^{-1}(z) \cap H_{i}$ are curves in $\mathbb{P}_{2}$

2) for all $i=1, \ldots, m$ the number of intersection points of $\pi^{-1}(0) \cap H_{i}$ and

$\left.\bigcup_{j=1, \ldots, m ; j \neq i}\left(\pi^{-1}(0) \cap H_{j}\right)\right)$ is at least three.

3) $\mathbb{P}_{2} \backslash \bigcup_{j=1, \ldots, m}\left(\pi^{-1}(0) \cap H_{j}\right)$ is hyperbolically embedded in $\mathbb{P}_{2}$.

Then $\mathbb{P}_{2} \backslash \bigcup_{j=1, \ldots, m}\left(\pi^{-1}(z) \cap H_{j}\right)$ is complete hyperbolic and hyperbolically embedded for all $z \in\left(\Delta_{s}\right)^{n}$ for some $s \leq t$.

Proof: In the terminology of [16], the $\pi^{-1}(0) \cap H_{i}$ form an absorbing $H$ stratification (cf. [16], p. 354 f.), for which we can apply Theorem 2.1 of [16]. Complete hyperbolicity follows from [12], p.36.

\section{Nonexistence of algebraic entire curves in generic complements}

In this section we prove that the complement of 3 generic quadrics, or of any 4 generic curves other than 4 lines, does not contain non-constant entire curves 
contained in an algebraic curve. Because of Corollary 3.1 this can be regarded as a statement of 'algebraic' hyperbolicity.

Let us first make precise what we mean by generic. The space of curves $\Gamma_{i}$ of degree $d_{i}$ in $\mathbb{P}_{2}$, which we define as the projectivized space of homogeneous polynomials of degree $d_{i}$, is a projective space of dimension $n_{i}=\frac{1}{2}\left(d_{i}+\right.$ $2)\left(d_{i}+1\right)-1$. Hence $\mathcal{C}\left(d_{1}, \ldots, d_{k}\right)=\prod_{i=1}^{k} \mathbb{P}_{n_{i}}$ is projective algebraic. In order to simplify notations we denote this space by $S$ in all what follows, and its elements by $s \in S$, and by $\Gamma_{i}(s)$ the curve given by the i-th component of $s \in S$.

Proposition 4.1 Let $S=\mathcal{C}(2,2,2)$ or $S=\mathcal{C}\left(d_{1}, \ldots, d_{k}\right)$ with $k \geq 4$ and $d=\sum_{i=1}^{k} d_{i} \geq 5$. Then there exists a proper algebraic variety $V \subset S$ st. for $s \in S \backslash V$ the following holds:

For any irreducible plane algebraic curve $A \subset \mathbb{P}_{2}$ the punctured Riemann surface $A \backslash \bigcup_{i=1}^{k} \Gamma_{i}(s)$ is hyperbolic, in particular any holomorphic map $f: \mathbb{C} \rightarrow$ $\mathbb{P}_{2} \backslash \bigcup_{i=1}^{k} \Gamma_{i}(s)$ with $f(\mathbb{C}) \subset A$ (which may also be reducible) is constant.

Proof: In order to define $V \subset S$ we list 5 conditions:

(1) All $\Gamma_{i}(s)$ are smooth (and of multiplicity one).

(2) The $\Gamma_{i}(s), i=1, \ldots, k$ intersect transversally, in particular no 3 of these intersect in one point.

(3) In the case of $\mathcal{C}(2,2,2)$ : For any common tangent line of two of the quadrics $\Gamma_{j}(s)$ which is tangential to these in points $P$ and $Q$ resp. the third quadric does not intersects the tangent in both points $P$ and $Q$.

(4) In the case of $\mathcal{C}\left(d_{1}, d_{2}, 1,1\right), d_{1}, d_{2} \geq 2$ : There does not exist a common tangent $L$ to $\Gamma_{1}(s)$ and $\Gamma_{2}(s)$ such that $L \cap \Gamma_{1}(s)=\{P\}$ and $L \cap \Gamma_{2}(s)=\{Q\}$ such that the lines $\Gamma_{3}(s)$ and $\Gamma_{4}(s)$ contain $P$ and $Q$ resp..

(5) In the case of $\mathcal{C}\left(d_{1}, 1,1,1\right)$ : There does not exist a tangent line $L$ at $\Gamma_{1}(s)$ with $L \cap \Gamma_{1}(s)=\{P\}$ such that $P$ is contained in one of the lines $\Gamma_{i}(s), i=2,3,4$ and $L$ contains the intersection points of the other two lines.

Define $V \subset S$ to be the set of those points $s \in S$ such that the $\Gamma_{i}(s)$ violates one of the above conditions. This set is clearly algebraic and not dense in $S$.

For intersections of at least five curves (2) implies that any irreducible algebraic curve $A$ intersects $\bigcup_{i=1}^{k} \Gamma_{i}(s)$ in at least three different points, which proves the claim.

Assume that there exists an irreducible algebraic curve $A \subset \mathbb{P}_{2}$ and $s \in S$ such that $A \backslash \bigcup_{i=1}^{k} \Gamma_{i}(s)$ is not hyperbolic. By condition (2) we know that $A \cap \bigcup_{i=1}^{k} \Gamma_{i}(s)$ consists of at least 2 points $P$ and $Q$. Moreover, $A$ cannot have 
a singularity at $P$ or $Q$ with different tangents, because $A$ had to be reducible in such a point, and $A \backslash \bigcup_{i=1}^{k} \Gamma_{i}(s)$ could be identified with an irreducible curve with at least three punctures. (This follows from blowing up such a point or considering the normalization).

So $A \cap \bigcup_{i=1}^{k} \Gamma_{i}(s)$ consists of exactly 2 points $P$ and $Q$ with simple tangents. We denote the multiplicities of $A$ in $P$ and $Q$ by $m_{P}$ and $m_{Q}$. Let $d_{0}=\operatorname{deg}(A)$. Then the inequality (cf. [4], p.117)

$$
m_{P}\left(m_{P}-1\right)+m_{Q}\left(m_{Q}-1\right) \leq\left(d_{0}-1\right)\left(d_{0}-2\right)
$$

implies

$$
m_{P}, m_{Q}<d_{0} \text { or } d_{0}=m_{P}=m_{Q}=1 \text {. }
$$

Let us now first treat the case $k=4$ : Each $\Gamma_{i}(s)$ contains exactly one of the points $P$ and $Q$. Let $\Gamma_{j}(s)$ and $\Gamma_{k}(s)$ resp. intersect $A$ in $P$ and $Q$ resp. not tangential, i.e. with tangents different from those of $A$ in these points. Let $d_{j}$ and $d_{k}$ be the degrees of these components. We compute intersection multiplicities according to [四, p.75

$$
m_{P}=I\left(P, A \cap \Gamma_{j}(s)\right)=d_{j} d_{0} \quad \text { and } \quad m_{Q}=I\left(Q, A \cap \Gamma_{k}(s)\right)=d_{k} d_{0} .
$$

Hence

$$
d_{0}=d_{j}=m_{P}=1 \text { and } d_{0}=d_{k}=m_{Q}=1 .
$$

In particular $A, \Gamma_{j}$ and $\Gamma_{k}$ are lines. These situations are excluded by (4) and (5).

Now let us treat the case of 3 quadrics. After a suitable enumeration of its components we may assume that $P \in \Gamma_{1}(s) \cap \Gamma_{2}(s)$ and $Q \in \Gamma_{3}(s)$. If $Q \notin \Gamma_{2}(s) \cup \Gamma_{1}(s)$ we are done, since then we may assume that $A$ is not tangential to $\Gamma_{2}(s)$, and again

$$
m_{P}=I\left(P, A \cap \Gamma_{2}(s)\right)=2 d_{0} .
$$

contradicts equation (3). So we may assume that $Q \in \Gamma_{2}(s) \cap \Gamma_{3}(s)$. Now $A$ has to be tangential to $\Gamma_{1}(s)$ in $P$ and to $\Gamma_{3}(s)$ in $Q$, otherwise we again get $m_{P}=2 d_{0}$ or $m_{Q}=2 d_{0}$ what contradicts equation (3). But then $\Gamma_{2}(s)$ is not tangential to $A$ in $P$ or $Q$, so we have

$$
m_{P}+m_{Q}=I\left(P, A \cap \Gamma_{2}(s)\right)+I\left(Q, A \cap \Gamma_{2}(s)\right)=2 d_{0}
$$

Again by equation (3) this is only possible if $m_{P}=m_{Q}=d_{0}=1$, but then we are in a situation which we excluded in condition (3), which is a contradiction. 


\section{Hyperbolicity of generic complements of at least four curves}

In this section we prove a result in the direction towards a generalized second main theorem. As a corollary we get a new proof of the fact that for any generic collection of four hypersurfaces $\Gamma_{j}, j=1, \ldots 4$ in $\mathbb{P}_{2}$ any entire curve $f: \mathbb{C} \rightarrow$ $\mathbb{P}_{2} \backslash \bigcup_{j=1}^{4} \Gamma_{j}$ has to be algebraically degenerate. This fact, combined with our result in the previous section implies the hyperbolicity of the complement of such a configuration.

Theorem 5.1 Let $S=\mathcal{C}\left(d_{1}, \ldots, d_{k}\right)$ with $k \geq 4, d=\sum_{i=1}^{k} d_{i} \geq 5$. Then there exists an algebraic variety $V \subset S$ such that for $s \in S \backslash V$ the following holds: Assume that $f: \mathbb{C} \rightarrow \mathbb{P}_{2} \backslash \bigcup_{j=1}^{3} \Gamma_{j}(s)$ is a non-constant holomorphic map. Then $\delta_{f}\left(\Gamma_{l}(s)\right)=0$ for $l=4, \ldots, k$. In particular, $f$ cannot miss any $\Gamma_{l}(s), l=4, \ldots, k$.

Proof: Let $V \subset S$ be defined like in the proof of Proposition 4.1, i.e. $s \in S \backslash V$, iff the conditions (1) to (5) given there are satisfied. Let $\Gamma_{i}(s)=\left\{P_{i}(s)=0\right\}$ for $i=1, \ldots, k$. For suitable powers $a_{j}$ we have because of condition (2) a morphism

$$
\Phi: \mathbb{P}_{2} \rightarrow \mathbb{P}_{2} ;\left[z_{0}: z_{1}: z_{2}\right] \rightarrow\left[P_{1}^{a_{1}}(s): P_{2}^{a_{2}}(s): P_{3}^{a_{3}}(s)\right]
$$

Since there exists no non-constant morphism on projective spaces, whose image is of lower dimension, for all $s \in S \backslash V$ the image $\Phi\left(\mathbb{P}_{2}\right)$ is not contained in an algebraic curve. From now on, we keep some $s \in S \backslash V$ fixed and drop the parameter $s$ for the rest of the proof. Furthermore let $\Phi\left(\Gamma_{4}\right)=\{Q=0\}$, where

$$
Q\left(w_{0}, w_{1}, w_{2}\right)=\sum_{i_{0}+i_{1}+i_{2}=e} a_{i_{0} i_{1} i_{2}} w_{0}^{i_{0}} w_{1}^{i_{1}} w_{2}^{i_{2}}
$$

so $\operatorname{deg} Q=e$. Finally, let $\Phi^{-1}\left(\Phi\left(\Gamma_{4}\right)\right)=\Gamma_{4} \cdot R$ be the decomposition of the inverse image curve of the curve $\Phi\left(\Gamma_{4}\right)$ in $\Gamma_{4}$ and the other components (which possibly may contain $\Gamma_{4}$ as well). Now the proof consists of 3 steps:

a) We have $a_{e 00} \neq 0, a_{0 e 0} \neq 0, a_{00 e} \neq 0$, i.e. the polynomial $Q$ contains the $e$-th powers of the coordinates:

We prove that indirectly, so without loss of generality we may assume that 
$a_{e 00}=0$. Then we have $Q([1: 0: 0])=0$, i.e. $[1: 0: 0] \in \Phi\left(\Gamma_{4}\right)$. So there exists a point $z \in \Gamma_{4}$ with $P_{1}(z) \neq 0, P_{2}(z)=0, P_{3}(z)=0$. But that means that the 3 curves $\Gamma_{2}, \Gamma_{3}$ and $\Gamma_{4}$ have a common point which contradicts our condition (2).

b) We show by using the Second Main Theorem that $\delta_{\Phi \circ f}\left(\Phi\left(\Gamma_{4}\right)\right)=0$ : Let $J=\left\{\left(\underline{i}=\left(i_{0}, i_{1}, i_{2}\right): a_{i_{0} i_{1} i_{2}} \neq 0\right\}\right.$ and $\kappa: J \rightarrow\{0,1, \ldots, p\}$ be an enumeration of $J$. Let $Q_{j}=w_{0}^{i_{0}} w_{1}^{i_{1}} w_{2}^{i_{2}}$ if $\kappa\left(\left(i_{0}, i_{1}, i_{2}\right)\right)=j$. Then by part a) the map

$$
\Psi: \mathbb{P}_{2} \rightarrow \mathbb{P}_{p} ;\left[w_{0}: w_{1}: w_{2}\right] \rightarrow\left[Q_{0}: \ldots: Q_{p}\right]
$$

is a morphism with components of degree $e=\operatorname{deg}(Q)$. The $p+2$ lines $L_{i}=$ $\left\{\xi_{i}=0\right\}, i=0, \ldots, p$ and $L=\left\{\sum_{\underline{i} \in J} a_{\underline{i}} \xi^{\kappa(\underline{i})}=0\right\}$ are in general position. Furthermore the map $\Psi \circ \Phi \circ f: \overline{\mathbb{C}} \rightarrow \mathbb{P}_{p}$ is linearly non degenerate: By Proposition 4.1, $f(\mathbb{C})$ is not contained in an algebraic curve, so especially not in an algebraic curve of the form $\sum_{\underline{i} \in J} b_{\underline{i}}\left(P_{1}^{a_{1}}\right)^{i_{0}}\left(P_{2}^{a_{2}}\right)^{i_{1}}\left(P_{3}^{a_{3}}\right)^{i_{2}}$, resulting from such a line in $\mathbb{P}_{p}$, unless the latter is identically zero. But this is impossible, since the map $\Phi$ is surjective. So we have by the Second Main Theorem:

$$
T(\Psi \circ \Phi \circ f, r) \leq N_{\Psi \circ \Phi \circ f}(L, r)+\sum_{i=0}^{p} N_{\Psi \circ \Phi \circ f}\left(L_{i}, r\right)+S(r)
$$

and by the First Main Theorem

$$
N_{\Psi \circ \Phi \circ f}(L, r) \leq T(\Psi \circ \Phi \circ f, r)+O(1)
$$

Observe that all $N_{\Psi \circ \Phi \circ f}\left(L_{i}, r\right)$ vanish. Together with Lemma 2.5 this yields, since $\operatorname{deg} Q=e$

$\delta_{\Phi \circ f}\left(\Phi\left(\Gamma_{4}\right)\right)=\liminf _{r \rightarrow \infty}\left(1-\frac{N_{\Phi \circ f}\left(\Phi\left(\Gamma_{4}\right), r\right)}{\operatorname{deg}(Q) T(\Phi \circ f, r)}\right)=\liminf _{r \rightarrow \infty}\left(1-\frac{N_{\Psi \circ \phi \circ f}(L, r)}{T(\Psi \circ \Phi \circ f, r)}\right)=0$

c) We finally show that $\delta_{f}\left(\Gamma_{4}\right)=0$ :

By equation (5) and Lemma 2.5 we have:

$$
\begin{gathered}
1=\limsup _{r \rightarrow \infty} \frac{N_{\Phi \circ f}\left(\Phi\left(\Gamma_{4}\right), r\right)}{\operatorname{deg}(Q) T(\Phi \circ f, r)}=\limsup _{r \rightarrow \infty} \frac{N_{f}\left(\Phi^{-1} \Phi\left(\Gamma_{4}\right), r\right)}{\operatorname{deg}(Q \circ \Phi) T(f, r)} \\
=\limsup _{r \rightarrow \infty} \frac{N_{f}\left(\Gamma_{4}, r\right)+N_{f}(R, r)}{\operatorname{deg}(Q \circ \Phi) T(f, r)}=\limsup _{r \rightarrow \infty} \frac{N_{f}\left(\Gamma_{4}, r\right)+N_{f}(R, r)}{\left(\operatorname{deg}\left(\Gamma_{4}\right)+\operatorname{deg}(R)\right) T(f, r)}
\end{gathered}
$$


or short:

$$
\limsup _{r \rightarrow \infty} \frac{N_{f}\left(\Gamma_{4}, r\right)}{T(f, r)}+\limsup _{r \rightarrow \infty} \frac{N_{f}(R, r)}{T(f, r)}=\operatorname{deg}\left(\Gamma_{4}\right)+\operatorname{deg}(R)
$$

By the First Main Theorem we have:

$$
\limsup _{r \rightarrow \infty} \frac{N_{f}(R, r)}{\operatorname{deg}(R) T(f, r)} \leq 1, \limsup _{r \rightarrow \infty} \frac{N_{f}\left(\Gamma_{4}, r\right)}{\operatorname{deg}\left(\Gamma_{4}\right) T(f, r)} \leq 1
$$

and hence with equation (6):

$$
\limsup _{r \rightarrow \infty} \frac{N_{f}\left(\Gamma_{4}, r\right)}{T(f, r)}=\operatorname{deg}\left(\Gamma_{4}\right), \text { i.e. } \delta_{f}\left(\Gamma_{4}\right)=0
$$

\section{Line systems through intersection points of three quadrics}

In this section, we study certain configurations of 18 lines associated to three smooth quadrics. These lines are needed in order to apply Value Distribution Theory to prove our main theorem in the next section.

Let $V^{\prime} \subset S=\mathcal{C}(2,2,2)$ be the algebraic variety defined by the conditions (1), (2), and (3) given in the Proof of Proposition 4.1, namely $s \in S \backslash V^{\prime}$, iff

(1) All $\Gamma_{i}(s)$ are smooth quadrics.

(2) The $\Gamma_{i}(s), i=1,2,3$ intersect transversally (in particular not all 3 intersect in one point)

(3) For any common tangent line of two of the quadrics $\Gamma_{j}(s)$ which is tangential to these in points $P$ and $Q$ resp. the third quadric does not intersects the tangent in $P$ and $Q$.

In order to prove our main theorem we will need one further condition of 'genericity' related to those 18 lines already mentioned above. For this condition it is quite not so obvious any more that it yields a quasiprojective set. We shall give an argument for this in Proposition 6.1. 
Let us first state the extra condition: Because of (2) any two of the three quadrics $\Gamma_{1}, \Gamma_{2}, \Gamma_{3}$ intersect in 4 distinct points $A_{1}, A_{2}, A_{3}, A_{4}$ which give rise to six lines

$$
\overline{A_{1} A_{2}}, \overline{A_{3} A_{4}} \text { and } \overline{A_{1} A_{3}}, \overline{A_{2} A_{4}} \text { and } \overline{A_{1} A_{4}}, \overline{A_{2} A_{3}} \text {. }
$$

So all three pairs of quadrics give rise to three sets $L_{12}(s), L_{13}(s)$ and $L_{23}(s)$ of six lines each, i.e. a collection $L(s)$ of 18 lines. We will show in the proof of Proposition 6.1 that as a consequence of (1) and (2) they are pairwise distinct.

Now our condition (4) reads:

(4) The 18 lines $L(s)$ intersect as follows: At any point of $\Gamma_{i}(s) \cap \Gamma_{j}(s), i \neq j$ there intersect exactly 3 of the 18 lines, and in every other point of $\mathbb{P}_{2}$ there intersect at most 2 of the 18 lines.

Now we have:

Proposition 6.1 Define $V \subset S$ to be the set of all $s \in S$ such that one of the conditions (1) to (4) is not satisfied. Then $V \subset S$ is a proper algebraic subset.

Proof: In order to prove the Proposition we use an argument which involves an elementary case of a Chow scheme.

We denote by $\mathbb{P}_{2}^{\vee}$ the space of all lines in $\mathbb{P}_{2}$. Look at the following rational map

$$
\begin{aligned}
\psi:\left(\mathbb{P}_{2}\right)^{4} & \rightarrow\left(\mathbb{P}_{2}^{\vee}\right)^{6} \\
\left(A_{1}, A_{2}, A_{3}, A_{4}\right) & \mapsto\left(A_{j} \wedge A_{k}\right)_{j<k}
\end{aligned}
$$

where the wedge product of two points is considered as an element of the dual projective space. This map descends to a rational map of symmetric spaces:

$$
\Psi: S^{4}\left(\mathbb{P}_{2}\right) \rightarrow S^{6}\left(\mathbb{P}_{2}^{\vee}\right)
$$

Over the complement of a proper algebraic subset it assigns to a set of four distinct points the configuration of six lines through these points.

Now we assign to any $s \in S \backslash V^{\prime}$ the tripel of sets $\left(\Gamma_{1}(s) \cap \Gamma_{2}(s), \Gamma_{1}(s) \cap\right.$ $\left.\Gamma_{3}(s), \Gamma_{2}(s) \cap \Gamma_{3}(s)\right)$, which amounts to a morphism

$$
\rho: S \backslash V^{\prime} \rightarrow\left(S^{4}\left(\mathbb{P}_{2}\right)\right)^{3} .
$$

Observe that $\Xi:=(\Psi)^{3} \circ \rho: S \backslash V^{\prime} \rightarrow\left(S^{6}\left(\mathbb{P}_{2}^{\vee}\right)\right)^{3}$ is a morphism. Now we can rephrase condition (4): 
Let $U \simeq \mathbb{C}^{3}$ and $W \simeq(U)^{6}$. Then we consider $W^{3}=\left\{\left(a_{j k}\right) \mid a_{j k} \in U ; j=\right.$ $1, \ldots, 6 ; k=1,2,3\}$ and look at the linear subspace $B \subset W^{3}$ defined by the condition that at least three components $a_{j_{1} k_{1}}, a_{j_{2} k_{2}}$ and $a_{j_{3} k_{3}}$ are linearly dependent where not all $k_{j}$ are the same. (We needn't care about the system of the six lines given by the four intersection points of two fixed quadrics, since they automatically have the desired intersection properties, because no three of the four intersection points of the two quadrics can be collinear.) Obviously $B$ descends to an algebraic set $\tilde{B} \in\left(S^{6}\left(\mathbb{P}_{2}^{\vee}\right)\right)^{3}$. Now (4) means for $s \in S \backslash V^{\prime}$ that $\Xi(s) \notin \tilde{B}$. The construction immediately implies that $V \backslash V^{\prime} \subset S \backslash V^{\prime}$ is algebraic, and since $V^{\prime} \subset S$ is algebraic, we have that $V=\overline{V \backslash V^{\prime}} \cup V^{\prime}$ is algebraic in $S$, where the closure here means the Zariski closure.

We have to show that $V \neq S$. The existence of an $s \in S \backslash V$ is proved by a deformation argument: We start with any $s \in S \backslash V^{\prime}$ (then the $\Gamma_{i}(s)$ are smooth and we have 12 different intersection points of two of the 3 quadrics each). It is easy to see that then we really have 18 different lines, otherwise 4 of the 12 intersection points of the 3 quadrics had to be contained in a line (because no three quadrics pass through a line). It follows from the construction, that this line would intersect one of the quadrics in 4 points, which is impossible.

Let $k=k(s)$ be the largest number of lines among the 18 lines (determined by the parameter $s$ ) which run through some point. Let $\nu_{k}=\nu_{k}(s)$ be the number of points in $\mathbb{P}_{2}$ which are contained in $k(s)$ of the lines. We will proceed now as follows: We observe that $k$ lines running through a point is a closed condition with respect to the classical topology of $S$. That means that in a neighborhood $U$ of a point $s_{0} \in S$ we have $k(s) \leq k\left(s_{0}\right)$, and at least $\nu_{k}(s) \leq \nu_{k}\left(s_{0}\right)$, if $k(s)=k\left(s_{0}\right)$. We will show that for some $s \in U$ actually $k(s)<k\left(s_{0}\right)$ or at least $\nu_{k}(s)<\nu_{k}\left(s_{0}\right)$, if $k(s)=k\left(s_{0}\right)$, as long as $k\left(s_{0}\right)>3$ or $k\left(s_{0}\right)=3$ but $\nu_{k}\left(s_{0}\right)>12$.

Iterating this procedure we are done if we can show: Consider the 18 lines in $L\left(s_{0}\right)$. If $k \geq 4$ take any of these intersection points where $k$ lines intersect (call it $T$ ), if $k=3$ take such an intersection point $T$ which is not intersection point of two of the quadrics. Then we can find $s \in S$ arbitrarily near to $s_{0}$ st. over $s$ the point $T$ 'breaks up' into intersection points of strictly less then $k\left(s_{0}\right)$ lines. But then $k(s)<k\left(s_{0}\right)$, or at least $k(s)=k\left(s_{0}\right)$ and $\nu_{k}(s)<\nu_{k}\left(s_{0}\right)$.

Let us now prove that: Take 3 of the lines running through $T$ over $s_{0}$ and denote them by $L_{1}, L_{2}, L_{3}$. Each of them is defined by construction by two of the intersection points of two of the 3 quadrics. Let $L_{1}$ be defined by such points $T_{1}, T_{2}$, let $L_{2}$ be defined by $T_{3}, T_{4}$ and let $L_{3}$ be defined by $T_{5}, T_{6}$. We 
may assume that no 3 of the 6 points $T_{1}, \ldots, T_{6}$ are equal to $T$ (this could only occur if $T$ is an intersection point of 2 of our 3 quadrics, but then $k \geq 4$ and we just have taken the 3 lines defined by $T$ and one other intersection point each, so we can choose a different line). So without loss of generality we may assume that $T_{1} \neq T \neq T_{2}$, and we have the following 3 possibilities for $L_{1}, L_{2}, L_{3}, T, T_{1}, \ldots, T_{6}$ :
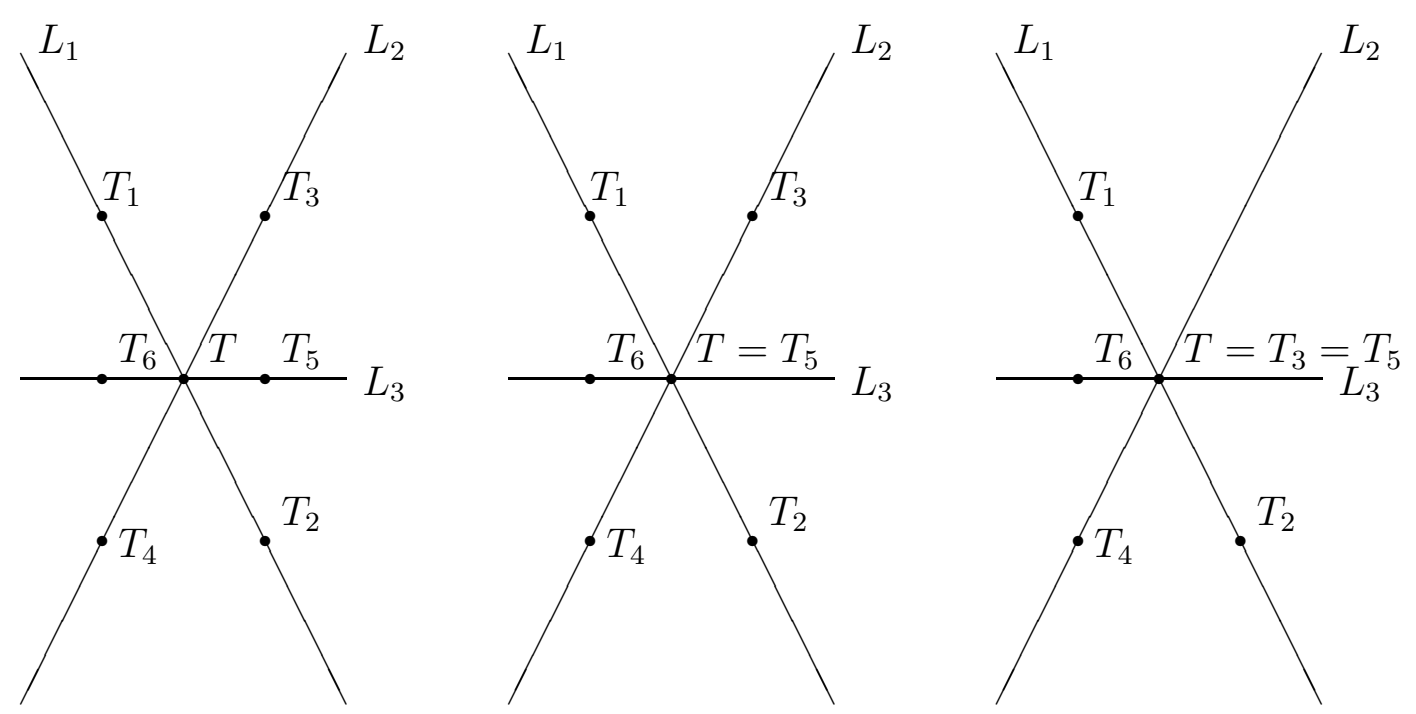

The point $T_{1}$ lies on 2 of the $\Gamma_{i}$, assume on $\Gamma_{1} \cap \Gamma_{2}$. Then at most 3 of the 4 or 5 different points in $\left\{T_{2}, \ldots, T_{6}\right\}$ can also be in $\Gamma_{1} \cap \Gamma_{2}$. So there exists one of them, call it $T_{0}$, which does lie on $\Gamma_{1} \cap \Gamma_{3}$ or $\Gamma_{2} \cap \Gamma_{3}$, assume on $\Gamma_{2} \cap \Gamma_{3}$. So at most 4 of the points $T_{2}, \ldots, T_{6}$ are contained in $\Gamma_{1}$. So we can 'move' $\Gamma_{1}$ while keeping these 4 points fixed and keeping $\Gamma_{2}$ and $\Gamma_{3}$ fixed. But that means that there is a non-constant variation of $s$ where we keep all of the points $T_{2}, \ldots, T_{6}$ fixed. Hence the lines $L_{2}$ and $L_{3}$ and their intersection point $T$ are kept fixed. We claim that for some small such variation the line $L_{1}$ does not pass any longer through $T$. If it would, it had to be fixed, since $T_{2}$ is kept fixed. By definition we have $T_{1} \in L_{1} \cap \Gamma_{1} \cap \Gamma_{2}$ and $L_{1} \cap \Gamma_{2}$ is a discrete set. Hence $T_{1}$ remains fixed. But that would mean that any quadric $\Gamma_{1}$ through the at most 4 of the fixed points $T_{2}, \ldots, T_{6}$ contained in $\Gamma_{1}$ must contain a fifth fixed point $T_{1}$. This is certainly a contradiction, since the space of plane quadrics is of dimension five. 
From any of the configurations of 18 lines in Proposition 6.1 we can pick 12 in general position:

Corollary 6.2 There exists an algebraic variety $V \subset S$ st. for all $s \in S \backslash V$ we have subsets of 12 of the 18 lines of Proposition 6.1 which are in general position.

Proof: For each pair $\Gamma_{i}(s), \Gamma_{j}(s), i \neq j$ we have constructed 3 pairs of lines (defined by equation (元)). Choose, for fixed $s \in S$, for each pair of quadrics two of these pairs of lines.

At last we prove the simple fact that the pairs of lines as defined in equation (7) are contained in the linear system spanned by the two quadrics.

Proposition 6.3 Let $\Gamma_{1}, \Gamma_{2}$ be two smooth quadrics intersecting in 4 different points $A_{1}, A_{2}, A_{3}, A_{4}$, and let the lines $L_{1}$ resp. $L_{2}$ be given by $A_{1}, A_{2}$ and $A_{3}, A_{4}$ resp. Then $L_{1} L_{2}$ is a degenerate quadric contained in the linear system spanned by $\Gamma_{1}$ and $\Gamma_{2}$, i.e. $L_{1} L_{2}=a \Gamma_{1}+b \Gamma_{2}$.

Proof: Look at the set $\mathcal{L}$ of all quadrics (possibly singular) which run through the 4 points $A_{1}, A_{2}, A_{3}, A_{4}$. Then $\mathcal{L}$ is a one dimensional linear system containing $L_{1} L_{2}$. Since it is one dimensional, it is spanned by any 2 of its elements, e.g. by $\Gamma_{1}$ and $\Gamma_{2}$.

\section{Hyperbolicity of generic complements of three quadrics}

We will prove:

Theorem 7.1 Let $V \subset S$ be the variety defined in Proposition 6.1. Let $s \in$ $S \backslash V$. Then the quasiprojective variety $\mathbb{P}_{2} \backslash \bigcup_{i=1}^{3} \Gamma_{i}(s)$ is complete hyperbolic and hyperbolically embedded.

Remark 7.2 The variety $S \backslash V$ is certainly not contained in an open subset of the space of all divisors of degree 6 whose complement in $\mathbb{P}_{2}$ is hyperbolic (cf. also [10]): Take any quadratic polynomials $P_{1}, P_{2}, P_{3}$ corresponding to some

$s \in S \backslash V$. Then with respect to suitable coordinates we have $P_{1}=z_{0}^{2}-z_{1} z_{2}$. Set $Q=\left(z_{1}^{6}+P_{1} \cdot F\right)$, where $F$ is an arbitrary polynomial of degree $4, P=P_{1} \cdot P_{2} \cdot P_{3}$, 
and $R_{t}=P+t \cdot Q, t \in \mathbb{C}$. Then the zero set of $R_{0}$ is just $\bigcup_{i=1}^{3} \Gamma_{i}(s)$. However, for $t \neq 0$ the intersection of $V\left(R_{t}\right)$ with the rational curve $V\left(P_{1}\right)$ consists only of the point $[0: 0: 1] \in \mathbb{P}_{2}$.

Proof of the Theorem: By Corollary 3.1 it is sufficient to show that there doesn't exist a non-constant entire curve $f: \mathbb{C} \rightarrow \mathbb{P}_{2} \backslash \bigcup_{i=1}^{3} \Gamma_{i}(s)$ of order at most 2 .

Assume there exists such a non-constant entire curve $f$. From Proposition 4.1 we know that $f$ is not algebraically degenerate.

For simplicity of notation we drop the $s$ in the rest of the proof. Furthermore we enumerate the 12 lines which we constructed in Proposition 6.1 and Corollary 6.2 as follows:

$L_{1} L_{2}$ and $L_{3} L_{4}$ are in the linear system of $\Gamma_{1}$ and $\Gamma_{2}$

$L_{5} L_{6}$ and $L_{7} L_{8}$ are in the linear system of $\Gamma_{1}$ and $\Gamma_{3}$

$L_{9} L_{10}$ and $L_{11} L_{12}$ are in the linear system of $\Gamma_{2}$ and $\Gamma_{3}$.

Let $\Gamma_{i}=\left\{P_{i}=0\right\}$ with a homogeneous polynomial $P_{i}$ of degree 2 .

The map $\Phi=\left[P_{1}: P_{2}: P_{3}\right]: \mathbb{P}_{2} \rightarrow \mathbb{P}_{2}$ is a morphism (because $\Gamma_{1} \cap \Gamma_{2} \cap \Gamma_{3}=$ $\emptyset)$. Furthermore the map $\Phi \circ f: \mathbb{C} \rightarrow \mathbb{P}_{2}$ again is an entire curve and the map $\Phi \circ f$ is again of finite order at most 2, because by Lemma 2.5 we have

$$
T(\Phi \circ f, r)=2 \cdot T(f, r)+O(1)
$$

Since $f$ misses the divisor $\Gamma_{1} \Gamma_{2} \Gamma_{3}$ the map $\Phi \circ f$ misses the divisors $\left\{z_{i}=\right.$ $0\}, i=1,2,3$ and hence by Lemma 2.3 we can write

$$
\Phi \circ f=\left[g_{0}: g_{1}: g_{2}\right]
$$

with

$$
g_{i}=e^{\alpha_{i} \xi^{2}+\beta_{i} \xi+\gamma_{i}} ; \alpha_{i}, \beta_{i}, \gamma_{i} \in \mathbb{C}
$$

where $g_{i}=\left(P_{i} \circ f\right) \cdot h ; h: \mathbb{C} \rightarrow \mathbb{C}^{*}$ are entire functions.

We may assume that not all three $\alpha_{j}$ are equal: Assume $\alpha_{1}=\alpha_{2}=\alpha_{3}$, then we can divide out the function $e^{\alpha_{1} \xi^{2}}$ and then compose the resulting functions with $\xi \mapsto \xi^{2}$, i.e. we may consider the function $\Phi \circ f\left(\xi^{2}\right)$. This map is again of order at most 2 and we have $g_{i}=e^{\beta_{i} \xi^{2}+\gamma_{i}}$. If now $\beta_{1}=\beta_{2}=\beta_{3}$, the map $\Phi \circ f$ would be constant, which is impossible, since $f$ is algebraically non degenerate. So we exclude the case $\alpha_{1}=\alpha_{2}=\alpha_{3}$ without loss of generality. 
The Ahlfors Lemma 2.4 allows the computation of some limits of characteristic functions: For $1 \leq i<j \leq 3$ :

$$
\lim _{r \rightarrow \infty} \frac{T\left(\left[P_{i} \circ f: P_{j} \circ f\right], r\right)}{r^{2}}=\lim _{r \rightarrow \infty} \frac{T\left(\left[g_{i}: g_{j}\right], r\right)}{r^{2}}=\frac{2\left|\alpha_{i}-\alpha_{j}\right|}{2 \pi}
$$

and

$$
\begin{gathered}
\lim _{r \rightarrow \infty} \frac{T\left(\left[P_{1} \circ f: P_{2} \circ f: P_{3} \circ f\right], r\right)}{r^{2}}=\lim _{r \rightarrow \infty} \frac{T\left(\left[g_{1}: g_{2}: g_{3}\right]\right)}{r^{2}} \\
=\frac{\left|\alpha_{1}-\alpha_{2}\right|+\left|\alpha_{1}-\alpha_{3}\right|+\left|\alpha_{2}-\alpha_{3}\right|}{2 \pi}
\end{gathered}
$$

hold. Now we want to relate the counting functions of the 12 lines to the characteristic functions used in equations ([10) and ([1]): We know that $L_{1} L_{2}$ is in the linear system of $\Gamma_{1}$ and $\Gamma_{2}$, i.e. $L_{1} L_{2}=a \Gamma_{1}+b \Gamma_{2}$ with $a, b \neq 0$ since $\Gamma_{1}$ and $\Gamma_{2}$ are smooth quadrics. We consider the map

$$
\left[P_{1} \circ f: P_{2} \circ f\right]: \mathbb{C} \rightarrow \mathbb{P}_{1} .
$$

Its image is not contained in a hyperplane in $\mathbb{P}_{1}$, i.e. a point, since $f$ is algebraically non degenerate. Furthermore the 3 divisors

$$
\left[z_{0}=0\right],\left[z_{1}=0\right],\left[a z_{0}+b z_{1}=0\right]
$$

are in general position in $\mathbb{P}_{1}$, i.e. distinct. The Second Main Theorem yields

$$
\begin{gathered}
T\left(\left[P_{1} \circ f: P_{2} \circ f\right], r\right) \leq N_{\left[P_{1} \circ f: P_{2} \circ f\right]}\left(\left[z_{0}=0\right], r\right) \\
\left.+N_{\left[P_{1} \circ f: P_{2} \circ f\right]}\left(\left[z_{1}=0\right], r\right)+N_{\left[P_{1} \circ f: P_{2} \circ f\right]}\left(a z_{0}+b z_{1}=0\right], r\right)+O(\log r)= \\
N_{f}\left(\left[P_{1}=0\right], r\right)+N_{f}\left(\left[P_{2}=0\right], r\right)+N_{f}\left(\left[a P_{1}+b P_{2}=0\right], r\right)+O(\log r)= \\
0+0+N_{f}\left(\left[L_{1} L_{2}=0\right], r\right)+O(\log r)=N_{f}\left(\left[L_{1}=0\right], r\right)+N_{f}\left(\left[L_{2}=0\right], r\right)+O(\log r)
\end{gathered}
$$

where $N_{f}\left(\left[P_{i}=0\right], r\right)=0$ because $f$ misses $\Gamma_{i}=\left[P_{i}=0\right]$, and where we identify the line $L_{i}$ with its defining equation, so that $\left[L_{i}=0\right]$ makes sense. On the other hand we have by the First Main Theorem

$$
N_{\left[P_{1} \circ f: P_{2} \circ f\right]}\left(\left[a z_{0}+b z_{1}=0\right], r\right) \leq T\left(\left[P_{1} \circ f: P_{2} \circ f\right], r\right)+O(1)
$$

and hence

$$
T\left(\left[P_{1} \circ f: P_{2} \circ f\right], r\right)=N_{f}\left(\left[L_{1}=0\right], r\right)+N_{f}\left(\left[L_{2}=0\right], r\right)+O(\log r)
$$


The corresponding equations hold for all other lines as well, i.e. we have:

$$
\begin{gathered}
T\left(\left[P_{1} \circ f: P_{2} \circ f\right], r\right)=N_{f}\left(\left[L_{1}=0\right], r\right)+N_{f}\left(\left[L_{2}=0\right], r\right)+O(\log r) \\
=N_{f}\left(\left[L_{3}=0\right], r\right)+N_{f}\left(\left[L_{4}=0\right], r\right)+O(\log r) \\
T\left(\left[P_{1} \circ f: P_{3} \circ f\right], r\right)=N_{f}\left(\left[L_{5}=0\right], r\right)+N_{f}\left(\left[L_{6}=0\right], r\right)+O(\log r) \\
=N_{f}\left(\left[L_{7}=0\right], r\right)+N_{f}\left(\left[L_{8}=0\right], r\right)+O(\log r) \\
T\left(\left[P_{2} \circ f: P_{3} \circ f\right], r\right)=N_{f}\left(\left[L_{9}=0\right], r\right)+N_{f}\left(\left[L_{10}=0\right], r\right)+O(\log r) \\
=N_{f}\left(\left[L_{11}=0\right], r\right)+N_{f}\left(\left[L_{12}=0\right], r\right)+O(\log r) .
\end{gathered}
$$

Since $f: \mathbb{C} \rightarrow \mathbb{P}_{2}$ is not linearly degenerate and the 12 lines $L_{1}, \ldots, L_{12}$ are in general position, we can again apply the Second Main Theorem and get

$$
9 \cdot T(f, r) \leq \sum_{i=1}^{12} N_{f}\left(\left[L_{i}=0\right], r\right)+O(\log r) .
$$

The equations (8), (12) and (13) imply

$$
\begin{gathered}
\frac{9}{2} \cdot T(\Phi \circ f, r)=9 \cdot T(f, r)+O(1) \leq \sum_{i=1}^{12} N_{f}\left(\left[L_{i}=0\right], r\right)+O(\log r) \\
=2 \cdot\left(T\left(\left[P_{1} \circ f: P_{2} \circ f\right], r\right)+T\left(\left[P_{1} \circ f: P_{3} \circ f\right], r\right)+T\left(\left[P_{2} \circ f: P_{3} \circ f\right], r\right)+O(\log r)\right.
\end{gathered}
$$

Hence together we have

$$
9 \cdot T(\Phi \circ f, r) \leq 4 \cdot\left(\sum_{1 \leq i<j \leq 3} T\left(\left[P_{i} \circ f: P_{j} \circ f\right], r\right)\right)+O(\log r) .
$$

We now divide equation (14) by $r^{2}$ and take $\lim _{r \rightarrow \infty}$. Using the equations (10) and (11) we obtain:

$9 \cdot \frac{\left|\alpha_{1}-\alpha_{2}\right|+\left|\alpha_{1}-\alpha_{3}\right|+\left|\alpha_{2}-\alpha_{3}\right|}{2 \pi} \leq 4 \cdot 2 \cdot \frac{\left|\alpha_{1}-\alpha_{2}\right|+\left|\alpha_{1}-\alpha_{3}\right|+\left|\alpha_{2}-\alpha_{3}\right|}{2 \pi}$.

This can only hold if $\alpha_{1}=\alpha_{2}=\alpha_{3}$, which is a contradiction. 


\section{Complements of two quadrics and a line}

In this section we need the following theorem of M. Green [6] (in degree $d=2$ ) which generalizes in a sense the classical Borel lemma.

Theorem 8.1 a) Let $g_{0}, g_{1}, g_{2}$ be entire holomorphic functions of finite order, $g_{1}$ and $g_{2}$ both nowhere vanishing. Assume that

$$
g_{0}^{2}+g_{1}^{2}+g_{2}^{2}=1
$$

Then the set

$$
\left\{1, g_{0}, g_{1}, g_{2}\right\}
$$

of holomorphic functions has to be linearly dependent.

b) Let $g_{0}$ and $g_{1}$ be entire holomorphic functions of finite order, $g_{1}$ nowhere vanishing. Assume that

$$
g_{0}^{2}+g_{1}^{2}=1
$$

Then $g_{0}$ and $g_{1}$ must be constant.

We consider the complement of three quadrics. We allow one of these to be also a double line. (The case, where one of the three quadrics degenerates to two distinct lines, i.e. two quadrics and two lines, has already been treated above).

Since in this section we work also with double lines we will distinguish between $\Gamma$ and $P$, where $\Gamma=V(P)$ (for simplicity reasons we didn't always do this in the previous sections).

Before we state the main result of this section, we observe that also some singular configurations of two quadrics in the projective plane can be treated by means of the generalized Borel lemma.

Proposition 8.2 Let $\Gamma_{j}=\left\{Q_{j}=0\right\} \subset \mathbb{P}_{2}, j=1,2$ be two smooth distinct quadrics, whose intersection consists of exactly one point. Then any holomorphic map $f: \mathbb{C} \rightarrow \mathbb{P}_{2} \backslash\left(\Gamma_{1} \cup \Gamma_{2}\right)$ of finite order has values in a quadric (which may degenerate to a double line) from the linear system spanned by $Q_{1}, Q_{2}$.

Proof. Let the common tangent line to $\Gamma_{1}$ and $\Gamma_{2}$ through the intersection point be defined by the linear equation $L=0$. One verifies immediately that

$$
L^{2}=a Q_{1}+b Q_{2}
$$


$a, b \neq 0$. Let $q_{j}$ be entire non-vanishing holomorphic functions, $j=1,2$ such that $q_{j}^{2}=Q_{j} \circ f$, and $q_{0}=L \circ f$. Then Theorem 8.1 b) implies that $Q_{1} \circ f=$ $c \cdot Q_{2} \circ f$.

Another case is the following.

Proposition 8.3 Let $\Gamma_{j}=\left\{Q_{j}=0\right\} \subset \mathbb{P}_{2}, j=1,2$ be two smooth distinct quadrics, which intersect exactly at two points tangentially. Then any holomorphic map $f: \mathbb{C} \rightarrow \mathbb{P}_{2} \backslash\left(\Gamma_{1} \cup \Gamma_{2}\right)$ of finite order has values in a quadric contained in the linear system spanned by $Q_{1}, Q_{2}$.

Proof. The linear system spanned by $Q_{1}$ and $Q_{2}$ contains $L^{2}$, where $L$ is the line through the two points of intersection. Using this the statement follows as above.

Theorem 8.4 Let $0 \neq Q_{j} \in \mathbb{C}\left[z_{0}, z_{1}, z_{2}\right], j=1,2,3$ be quadratic polynomials, where either all $Q_{j}$ are irreducible or all but one which may be a square of a linear function. Let $\Gamma_{j} \subset \mathbb{P}_{2}$ be the zero-sets. Assume

(1) no more than two of these intersect at one point,

(2) no tangent to a smooth quadric $\Gamma_{j}$ at a point of intersection with some other $\Gamma_{k}$ contains a further intersection point of the curves $\Gamma_{l}$,

(3) there exists a linear combination of the $Q_{j}$ which is a square:

$$
\sum_{j=1}^{3} a_{j} Q_{j}=P^{2}, \quad P \in \mathbb{C}\left[z_{0}, z_{1}, z_{2}\right],
$$

where at least two coefficients $a_{j}$ are different from zero.

Then any holomorphic map

$$
f: \mathbb{C} \rightarrow \mathbb{P}_{2} \backslash \bigcup_{j=1}^{3} \Gamma_{j}
$$

has values in a quadric (which may be degenerate to a double line).

We call a holomorphic map $\mathbb{C} \rightarrow \mathbb{P}_{2}$ linearly or quadratically degenerate, if its values are contained in a line or a (possibly degenerate) quadric resp.. 
Corollary 8.5 Let $\Gamma_{j}=V\left(Q_{j}\right) \subset \mathbb{P}_{2}, j=2,3$ be smooth quadrics and $\Gamma_{1}=$ $L_{1}=V\left(Q_{1}\right) \subset \mathbb{P}_{2}$ a line, where $Q_{1}$ is the square of a linear polynomial, and let the assumptions of 8.4 be satisfied.

(1) The quasiprojective variety $\mathbb{P}_{2} \backslash \bigcup_{j=1}^{3} \Gamma_{j}$ is Brody-hyperbolic, unless there exists a smooth quadric or a line $\Gamma$ such that after choosing the notation accordingly ( $p, q$ distinct points):

(a) $\Gamma \cap \Gamma_{2}=\{p, q\}, \Gamma \cap \Gamma_{3}=\{p\}, \Gamma \cap L_{1}=\{q\}$

(b) $\Gamma \cap \Gamma_{2}=\{p\}, \Gamma \cap \Gamma_{3}=\{p\}, \Gamma \cap L_{1}=\{q\}$

(c) $\Gamma \cap \Gamma_{2}=\{p\}, \Gamma \cap \Gamma_{3}=\{q\}, \Gamma \cap L_{1}=\{p\}$

(d) $\Gamma \cap \Gamma_{2}=\{p\}, \Gamma \cap \Gamma_{3}=\{q\}, \Gamma \cap L_{1}=\{p, q\}$

(2) The quasiprojective variety $\mathbb{P}_{2} \backslash \bigcup_{j=1}^{3} \Gamma_{j}$ is complete hyperbolic and hyperbolically embedded, unless

(e) at least two of the $\Gamma_{j}$ are tangent to each other at some point,

(f) there exists a smooth quadric, which has only one point of intersection with each of $\Gamma_{2}$ and $\Gamma_{3}$ with both of these points contained in $\Gamma_{1}$,

(g) There exists a tangent to one of $\Gamma_{2}$ and $\Gamma_{3}$ at a point of intersection with $\Gamma_{1}$ which is tangent to the other smooth quadric.

We introduce the following polynomials which will take care of a necessary elimination process in the proof of 8.4 .

Definition 8.6 Let the homogeneous polynomial $R_{j}\left(y_{0}, \ldots, y_{j}\right) \in \mathbb{C}\left[y_{0}, \ldots, y_{j}\right]$ of degree $2^{j-1}$ be defined by the equation

$$
R_{j}\left(x_{0}^{2}, \ldots, x_{j}^{2}\right)=\prod_{\left(\epsilon_{1}, \ldots, \epsilon_{j}\right) \in\{1,-1\}^{j}}\left(x_{0}+\epsilon_{1} x_{1}+\ldots+\epsilon_{j} x_{j}\right) .
$$

For later applications we need some properties of the $R_{j}$ : 
Lemma 8.7 a) $R_{2}(x, y, z)=x^{2}+y^{2}+z^{2}-2 x y-2 x z-2 y z$

b) Let $a, b, c \in \mathbb{C}$. Then

$$
S(x, y, z):=R_{3}(a x+b y+c z, x, y, z)
$$

has the following properties:

1) The coefficient of $x^{4}$ equals $(a-1)^{4}$.

2) The coefficient of $x^{2} y^{2}$ equals $2\left(3 a^{2}(b-1)^{2}-2 a(b-1)(3 b+1)+3 b^{2}+2 b+3\right)$. In particular, if the coefficient of $y^{4}$ vanishes, the coefficient of $x^{2} y^{2}$ equals 16.

3) Assume that all coefficients of forth powers in $S$ vanish. Then

$$
S(x, y, z)=16\left(x^{2} y^{2}+x^{2} z^{2}+y^{2} z^{2}\right)-32\left(x^{2} y z+x y^{2} z+x y z^{2}\right) .
$$

We omit the computational proof.

Proof of Theorem 8.4. Since the map $f$ has no values in the given quadrics $\Gamma_{j}$, there exist entire holomorphic functions $q_{j}, j=1,2,3$ such that $q_{j}^{2}=Q_{j} \circ f$. If we put then $q_{0}=P \circ f$ and $g_{j}=q_{j} / q_{3}$ for $j=0,1,2$. We apply the generalized Borel lemma (Theorem 8.1): If one of the $a_{j}$ vanishes, we get immediately quadratic degeneracy from part b) of this theorem.

So from now on we assume that all $a_{j}$ are non-zero. Thereom $8.1 \mathrm{a}$ ) implies that the set of functions $\left\{1, g_{0}, g_{1}, g_{2}\right\}$ is linearly dependent, i.e. $\left\{q_{0}, \ldots, q_{3}\right\}$ has this property.

Let

$$
\sum_{j=0}^{3} \alpha_{j} q_{j}=0, \quad \text { not all } \alpha_{j}=0,
$$

and let $R=R_{3}$ be the polynomial of 8.6. It has been chosen in a way such that $R\left(\alpha_{0}^{2} q_{0}^{2}, \ldots, \alpha_{3}^{2} q_{3}^{2}\right)=0$. The assumption (16) means that $q_{0}^{2}=a_{1} q_{1}^{2}+a_{2} q_{2}^{2}+a_{3} q_{3}^{2}$. Now the curve defined by the equation

$$
\tilde{R}\left(z_{0}, z_{1}, z_{2}\right)=R\left(\alpha_{0}^{2}\left(a_{1} Q_{1}+a_{2} Q_{2}+a_{3} Q_{3}\right), \alpha_{1}^{2} Q_{1}, \alpha_{2}^{2} Q_{2}, \alpha_{3}^{2} Q_{3}\right)=0
$$

contains the image of $f$ and is of degree at most eight. We have to show that $\tilde{R}$ is not identically zero. Otherwise, since $\left(Q_{1}, Q_{2}, Q_{3}\right)$ defines a morphism, i.e. an epimorphism $Q: \mathbb{P}_{2} \rightarrow \mathbb{P}_{2}$, the polynomial $R\left(\alpha_{0}^{2}\left(a_{1} y_{1}^{2}+a_{2} y_{2}^{2}+\right.\right.$ 
$\left.\left.a_{3} y_{3}^{2}\right), \alpha_{1}^{2} y_{1}^{2}, \alpha_{2}^{2} y_{2}^{2}, \alpha_{3}^{2} y_{3}^{2}\right) \in \mathbb{C}\left[y_{1}, y_{2}, y_{3}\right]$ would be the zero polynomial. The definition of $R$ would imply that $\alpha_{0}^{2}\left(a_{1} y_{1}^{2}+a_{2} y_{2}^{2}+a_{3} y_{3}^{2}\right)=\left(\sum_{1}^{3} \delta_{j} \alpha_{j} y_{j}\right)^{2}$ for certain $\delta_{j}= \pm 1$. Thus at least two of $\alpha_{1}, \alpha_{2}, \alpha_{3}$ must vanish. However, by assumption, the $a_{j}$ are different from zero. From this fact it follows immediately that all $\alpha_{j}=0$, which is a contradiction. We have shown that $f(\mathbb{C})$ is contained in an algebraic curve of degree at most eight which is defined by a polynomial of degree four in $Q_{1}, Q_{2}, Q_{3}$.

Before we proceed with the proof of Theorem 8.4, we give an application of the classical Borel Lemma. Let $\mathbb{P}_{2}=\left\{z_{0}, z_{1}, z_{2}\right\}$, and $H_{j}=\left\{z_{j}=0\right\}$ be the coordinate hyperplanes.

Remark 8.8 Let $f: \mathbb{C} \rightarrow \mathbb{P}_{2} \backslash\left(H_{0} \cup H_{1} \cup H_{2}\right)$ be a holomorphic map. Assume that $f$ is algebraically degenerate, i.e. its values are contained in an algebraic curve $C$. Then $f(\mathbb{C}) \subset C^{\prime}$, where $C^{\prime}$ is the zero-set of a polynomial of the form $z_{0}^{k}-\beta z_{1}^{l} z_{2}^{m}, \beta \neq 0, k, l+m \leq \operatorname{deg}(C)$ (after a suitable reordering of indices).

Proof. Let $C$ be the zero-set of some polynomial $P\left(z_{0}, z_{1}, z_{2}\right)$. Denote by $f_{j}$ the components of $f$. The classical lemma of Borel, applied to the monomials in the expansion of $P$, implies that there exist at least two such monomials, which are proportional after composing with $f$. Thus $f_{0}^{r} f_{1}^{s} f_{2}^{t}=\beta f_{0}^{u} f_{1}^{v} f_{2}^{w}$ for some $\beta \neq 0$.

(The statement of the Lemma has an obvious generalization to $\mathbb{P}_{n}$.)

An immediate consequence of Theorem 8.4, as far as we have proved is yet, and of Remark 8.8 is, since all $Q_{j} \circ f$ have no zeroes,

Lemma 8.9 Given the assumptions of 8.4, the image $f(\mathbb{C})$ is contained in a curve of the form

$$
Q_{u}^{k}-\alpha Q_{v}^{l} Q_{w}^{m}=0, \quad \alpha \neq 0,
$$

where $\{u, v, w\}=\{1,2,3\}$, and $k, l+m \leq 4$.

We note the following fact:

Lemma 8.10 Let $f: \mathbb{C} \rightarrow \mathbb{P}_{2}$ be as above.

(1) Let $f(\mathbb{C})$ be contained in the zero-set

$$
V\left(Q_{1}^{k_{1}} Q_{2}^{k_{2}} Q_{3}^{k_{3}}-\alpha Q_{1}^{l_{1}} Q_{2}^{l_{2}} Q_{3}^{l_{3}}\right),
$$

with $\sum k_{j}=\sum l_{j}=4$, and $k_{j}=l_{j}$ for at least one $j$, or 
(2) let $f(\mathbb{C})$ be contained in both zero-sets

$$
V\left(Q_{1}^{k_{1}} Q_{2}^{k_{2}} Q_{3}^{k_{3}}-\alpha Q_{1}^{l_{1}} Q_{2}^{l_{2}} Q_{3}^{l_{3}}\right)
$$

and

$$
V\left(Q_{1}^{m_{1}} Q_{2}^{m_{2}} Q_{3}^{m_{3}}-\beta Q_{1}^{n_{1}} Q_{2}^{n_{2}} Q_{3}^{n_{3}}\right)
$$

with $\sum k_{\nu}=\sum l_{\nu}=\sum m_{\nu}=\sum n_{\nu}=4$ such that the vector

$$
\left(k_{1}-l_{1}, k_{2}-l_{2}, k_{3}-l_{3}\right)
$$

is not a rational multiple of the vector

$$
\left(m_{1}-n_{1}, m_{2}-n_{2}, m_{3}-n_{3}\right) \text {. }
$$

Then $f(\mathbb{C})$ is contained in a quadric curve, which is a member of the linear system generated by two of the quadrics $Q_{j}$.

We call the monomials $Q_{1}^{k_{1}} Q_{2}^{k_{2}} Q_{3}^{k_{3}}$ and $Q_{1}^{l_{1}} Q_{2}^{l_{2}} Q_{3}^{l_{3}}$ satisfying (19) equivalent with respect to $f$. Proof. We can eliminate one of the $Q_{j}$, say $Q_{1}$, and obtain that $f(\mathbb{C})$ is contained in $V\left(Q_{2}^{r}-\gamma Q_{3}^{r}\right)$ for some integer $r$, and $\gamma \in \mathbb{C}$, because the $Q_{j} \circ f$ have no zeroes. Taking roots we find that $f(\mathbb{C}) \subset V\left(\sigma Q_{2}-\tau Q_{3}\right)$, $\sigma, \tau \in \mathbb{C}$ not both equal to zero.

We return to the proof of Theorem 8.4, and consider under which conditions the above lemma can be applied. Let the situation of Theorem 8.4 be given. Denote by

$$
T\left(Q_{1}, Q_{2}, Q_{3}\right)=R\left(\alpha_{0}^{2}\left(a_{1} Q_{1}+a_{2} Q_{2}+a_{3} Q_{3}\right), \alpha_{1}^{2} Q_{1}, \alpha_{2}^{2} Q_{2}, \alpha_{3}^{2} Q_{3}\right)
$$

the polynomial of (18).

We know that $T$ is not the zero-polynomial. We already reduced the proof of Theorem 8.4 to the case, where all $a_{j}$ in (16) are different from zero.

First part: Let all $\alpha_{j} \neq 0$. Thus we can (after normalizing these constants to 1) apply Lemma 8.10 .

First case: We claim that the conditions of Lemma 8.10 (2) are satisfied, if at least two of the coefficients of $Q_{j}^{4}$ in $T$, say those of $Q_{1}^{4}$ and $Q_{2}^{4}$, are different from zero.

If $Q_{1}^{4}$ and $Q_{2}^{4}$ are equivalent, $Q_{1} \circ f$ is a constant multiple of $Q_{2} \circ f$, and $f$ is quadratically degenerate. 
Otherwise there exist exponents $\left(r_{1}, r_{2}, r_{3}\right),\left(s_{1}, s_{2}, s_{3}\right)$ of $Q_{j}$ that match $(4,0,0)$ and $(0,4,0)$ resp. in the sense of (20) and (21) resp. because of the classical Borel Lemma. Assume that the assumptions of Lemma 8.10 (2) are not fulfilled, so there exists a rational number $c$ such that

$$
(4,0,0)-\left(r_{1}, r_{2}, r_{3}\right)=c\left((0,4,0)-\left(s_{1}, s_{2}, s_{3}\right)\right) .
$$

Since $\left(r_{1}, r_{2}, r_{3}\right) \neq(4,0,0)$ we have $r_{1}<4$, thus $s_{1}>0$ and $c<0$. Now $0 \geq-r_{3}=c\left(-s_{3}\right) \geq 0$ implies $r_{3}=s_{3}=0$, so we can apply Lemma 8.10 (1).

Second case: The next case to consider is, where exactly one forth power occurs, say $Q_{1}^{4}$. According to Lemma 8.7 2), the coefficient of $Q_{1}^{2} Q_{2}^{2}$ in $T$ must be different from zero.

Assume first that $Q_{1}^{4}$ is equivalent to $Q_{1}^{2} Q_{2}^{2}$ with respect to $f$. Then Lemma 8.10 (1) is applicable. If these monomials are not equivalent, we have some (non trivial) relations $Q_{1}^{4} \sim Q_{1}^{r_{1}} Q_{2}^{r_{2}} Q_{3}^{r_{3}}$ and $Q_{1}^{2} Q_{2}^{2} \sim Q_{1}^{s_{1}} Q_{2}^{s_{2}} Q_{3}^{s_{3}}$. If the assumptions of Lemma 8.10 (2) would not hold, we had

$$
(4,0,0)-\left(r_{1}, r_{2}, r_{3}\right)=c\left((2,2,0)-\left(s_{1}, s_{2}, s_{3}\right)\right)
$$

for some $0 \neq c \in \mathbb{Q}$ and $0 \leq r_{j}, s_{j} \leq 4, \sum r_{j}=\sum s_{j}=4$. For $r_{3}=0$ Lemma 8.10 (1) could be applied. Only $0<r_{3} \leq 4$ is left; in particular $-r_{3}=c\left(-s_{3}\right)$ implies $c>0, s_{3}>0$. Now $r_{1} \neq 4$. Thus $4-r_{1}=c\left(2-s_{1}\right)$ gives $s_{1}=0$ or $s_{1}=1$. Furthermore $-r_{2}=c\left(2-s_{2}\right)$ holds. Again $r_{2}=0$ makes 8.10 (1) applicable so that we are left with $s_{2}=3$ or $s_{2}=4$. Thus $\left(s_{1}, s_{2}, s_{3}\right)=(0,3,1)$. Hence $f$ has values in the quartic curve $Q_{1}^{2}-\gamma Q_{2} Q_{3}=0$ for some $\gamma \in \mathbb{C}$.

Let $C$ be the curve $V\left(Q_{1}^{2}-\gamma Q_{2} Q_{3}\right)$. We note first that

$$
C \cap\left(\Gamma_{1} \cup \Gamma_{2} \cup \Gamma_{3}\right)=\Gamma_{1} \cap\left(\Gamma_{2} \cup \Gamma_{3}\right)
$$

The case, where two smooth quadrics $\Gamma_{j}$ intersect in exactly one point, yields immediately quadratic degeneracy by Proposition 8.2, and we are done. If one of the $\Gamma_{j}$ is a line, it cannot be tangent to both of the further given smooth quadrics - this is also excluded by assumption (2). Thus $C \cap\left(\Gamma_{1} \cup \Gamma_{2} \cup \Gamma_{3}\right)$ consists of at least three points. As $f(\mathbb{C})$ is contained in $C \backslash\left(\Gamma_{1} \cup \Gamma_{2} \cup \Gamma_{3}\right)$, the curve $C$ cannot be irreducible unless $f$ is constant. We are left with the case where $C$ decomposes into a line $l$ and a cubic. We have $C \cap \Gamma_{1}=\Gamma_{1} \cap\left(\Gamma_{2} \cup \Gamma_{3}\right)=$ $C \cap\left(\Gamma_{1} \cap\left(\Gamma_{2} \cup \Gamma_{3}\right)\right)$, which implies $l \cap \Gamma_{1}=\left(l \cap \Gamma_{2}\right) \cup\left(l \cap \Gamma_{3}\right)$. This equality means that $l \neq \Gamma_{1}$ and that $l \cap \Gamma_{1}$ consists of two distinct points $p^{\prime}$ and $p^{\prime \prime}$, 
(since no more than two of the $\Gamma_{j}$ pass through a point). Let $l \cap \Gamma_{2}=\left\{p^{\prime}\right\}$ and $l \cap \Gamma_{3}=\left\{p^{\prime \prime}\right\}$. This means that $l$ is at least tangent to one of the smooth quadrics and passes through one further intersection point of the $\Gamma_{j}$. This was excluded by assumption (2).

Third case: Assume finally that all coefficients of $Q_{j}^{4}$ in $T$ vanish. According to Lemma 8.73 ) the non-zero monomials in $T$ are $Q_{j}^{2} Q_{k}^{2}, j \neq k$ and $Q_{j}^{2} Q_{k} Q_{l}$, where $(j, k, l)$ run through all cyclic permutations of $(1,2,3)$. We pick $Q_{1}^{2} Q_{2}^{2}$ and check to which of the monomials it can be equivalent with respect to $f$. Lemma 8.10 (1) is directly applicable to all possible cases but $Q_{1}^{2} Q_{2}^{2} \sim Q_{1} Q_{2} Q_{3}^{2}$ which implies $Q_{1} Q_{2} \sim Q_{3}^{2}$. This case was already treated.

The claim is now shown under the assumption that all $\alpha_{j}$ are different from zero.

If two or more of the $\alpha_{j}$ vanish, the claim is already clear from (17): We then get the equation $\alpha_{j} q_{j}=-\alpha_{k} q_{k}$, which, after squaring both sides, yields us quadratic degeneracy immediately, or if $q_{0}$ is involved, by using that at least two of the $a_{i}$ are not zero. The remaining case is, where exactly one $\alpha_{j}=0$. Here we use $R_{2}$ from 8.6 and arrive at a polynomial $U\left(y_{1}, y_{2}, y_{3}\right)$ of degree two, such that $f(\mathbb{C})$ is contained in the zero-set of $U\left(Q_{1}, Q_{2}, Q_{3}\right)$. Again Borel's lemma is applied to its monomials. A non-empty subset of $\left\{Q_{1}^{2}, Q_{2}^{2}, Q_{3}^{2}, Q_{1} Q_{2}, Q_{1} Q_{3}, Q_{2} Q_{3}\right\}$ has to be divided into sets of $f$-equivalent polynomials. In the view of 8.10 (1) the only case to remain is $Q_{j}^{2} \sim Q_{k} Q_{l}$ where $(j, k, l)$ is a cyclic permutation of $(1,2,3)$. This case was treated above.

In the sequel we treat the case of the complement of two plane quadrics and a line and the case of three Fermat quadrics. We show that

Theorem 8.11 There exist

(a) a quasiprojective set $V \subset \mathcal{C}(2,2,1)$ of codimension one and

(b) an open, non-empty subset $U \subset \mathcal{C}(2,2,1)$ containing $V$

such that for all $s \in U$ the space $\mathbb{P}_{2} \backslash \Gamma(s)$ is complete hyperbolic and hyperbolically embedded.

Proof. The set $V$ will be constructed in a such a way that the configurations $\Gamma(s)$ for $s \in V$ satisfy the conditions of Proposition 3.2 so that (b) will follow from the first statement.

Let $\mathbb{P}_{2}=\left\{\left[z_{0}: z_{1}: z_{2}\right]\right\}$ and 


$$
\begin{aligned}
l & =c_{0} z_{0}+c_{1} z_{1}+c_{2} z_{2} \\
Q_{0} & =l^{2} \\
Q_{j} & =\sum_{k=0}^{2} a_{j k} z_{k}^{2}+b_{j 0} z_{0} z_{1}+b_{j 1} z_{0} z_{2}+b_{j 2} z_{1} z_{2}
\end{aligned}
$$

for $j=1,2$.

We include $Q_{0}$ in this notation and compute $a_{0 k}, b_{0 k}$ in terms of $c_{l}$. We shall discuss, when (16) holds for these.

Let $A=\left(a_{j k}\right)$ and $B=\left(b_{j k}\right)$. Let $\hat{A}$ be the adjoint matrix of $A$, i.e. $\hat{A} \cdot A=$ $\operatorname{det}(A) E$. For $\kappa^{2}, \lambda^{2}, \mu^{2} \in \mathbb{C}$ we consider the following linear combination

$$
\left(\kappa^{2}, \lambda^{2}, \mu^{2}\right) \cdot\left(\operatorname{det}(A)\left(\begin{array}{c}
z_{0}^{2} \\
z_{1}^{2} \\
z_{2}^{2}
\end{array}\right)+\hat{A} B \cdot\left(\begin{array}{c}
z_{0} z_{1} \\
z_{0} z_{2} \\
z_{1} z_{2}
\end{array}\right)\right)=\left(\kappa^{2}, \lambda^{2}, \mu^{2}\right) \cdot \hat{A} \cdot\left(\begin{array}{c}
Q_{0} \\
Q_{1} \\
Q_{2}
\end{array}\right)
$$

Looking at the left hand side one verifies that this expression is a square of a linear polynomial, if and only if the following equation holds:

$$
\left(\kappa^{2}, \lambda^{2}, \mu^{2}\right) \cdot \hat{A} B=2 \operatorname{det}(A)(\kappa \lambda, \kappa \mu, \lambda \mu)
$$

We set $a=\left(a_{j k}\right)_{j>0} \in \mathbb{C}^{6}, b=\left(b_{j k}\right)_{j>0} \in \mathbb{C}^{6}$, and $c=\left(c_{l}\right) \in \mathbb{C}^{3}$. So $A, B$ and $\hat{A}$ are now given in terms of $a, b, c$. We define $M \subset \mathbb{P}_{2} \times \mathbb{C}^{3} \times \mathbb{C}^{6} \times \mathbb{C}^{6}$ to be the set of all points $([\kappa: \lambda: \mu], c, a, b)$ for which (26) holds.

For all $m \in M$ the inequality $\operatorname{dim}_{m} M \geq 14$ holds, since (26) consists of three equations in $\kappa, \lambda, \mu, A, \hat{A}, B$ and hence in $\kappa, \lambda, \mu, a, b, c$. Consider the canonical projection pr: $\mathbb{P}_{2} \times \mathbb{C}^{3} \times \mathbb{C}^{6} \times \mathbb{C}^{6} \rightarrow \mathbb{C}^{3} \times \mathbb{C}^{6} \times \mathbb{C}^{6}$. Let $c_{0}=$ $(1,0,0), a_{0}=\left(\begin{array}{lll}0 & 1 & 0 \\ 0 & 0 & 1\end{array}\right)$, and $b \in \mathbb{C}^{6}$ arbitrary. Then we calculate that $\left(\mathbb{P}_{2} \times\left\{\left(c_{0}, b, a_{0}\right)\right\}\right) \cap M$ is zero-dimensional.

In Example 8.12 we shall give an explicit example of a point $m_{0}=\left(\left[\kappa_{0}\right.\right.$ : $\left.\left.\lambda_{0}: \mu_{0}\right], c_{0}, b_{0}, a_{0}\right)$ which is contained in such a zero dimensional set, where $\kappa_{0}, \lambda_{0}, \mu_{0} \neq 0$. Denote by $M_{0} \subset M$ an irreducible component of $M$ containing $m_{0}$. Now $\operatorname{pr}\left(M_{0}\right) \subset \mathbb{C}^{3} \times \mathbb{C}^{6} \times \mathbb{C}^{6}$ is algebraic and at least of dimension 14 , because the fiber is zero dimensional. (One can check easily that $\operatorname{pr}\left(M_{0}\right) \neq$ $\left.\mathbb{C}^{3} \times \mathbb{C}^{6} \times \mathbb{C}^{6}\right)$. 
Let

$$
\left(\kappa^{2}, \lambda^{2}, \mu^{2}\right) \cdot \hat{A}=(\phi, \psi, \chi),
$$

and $N=V(\phi \cdot \psi \cdot \chi \cdot \operatorname{det}(A)) \subset M$. Observe $M_{0} \backslash N \neq \emptyset$, since $m_{0} \notin N$ (what can be checked easily). Let $V^{\prime} \subset \mathbb{C}^{3} \times \mathbb{C}^{6} \times \mathbb{C}^{6}$ be the quasi projective hypersurface $V^{\prime}=\operatorname{pr}\left(M_{0}\right) \backslash \operatorname{pr}(N) \subset \operatorname{pr}\left(M_{0} \backslash N\right)$, which is not empty: The fiber of $\operatorname{pr} \mid M_{0}$ at $m_{0}$ is of dimension zero, hence $\operatorname{dim}(\operatorname{pr}(N)) \leq \operatorname{dim} N<\operatorname{dim} M_{0}=\operatorname{dim}\left(\operatorname{pr}\left(M_{0}\right)\right)$.

By means of the assignment $\mathbb{C}^{3} \times \mathbb{C}^{6} \times \mathbb{C}^{6} \ni(c, b, a) \mapsto\left(l, Q_{1}, Q_{2}\right) \in$ $\mathbb{C}^{3} \times \mathbb{C}^{6} \times \mathbb{C}^{6}$ we associate to any point of $\operatorname{pr}\left(M_{0}\right)$ a triple consisting of one linear and two quadratical polynomials. Now $\operatorname{pr}\left(M_{0}\right)$ as well as $\operatorname{pr}(N)$ are invariant under the canonical action of $\left(\mathbb{C}^{*}\right)^{3}$, given by multiplication of $l, Q_{1}$, $Q_{2}$ by elements of $\mathbb{C}^{*}$. This follows from the original definition of $M$ and $N$ (the existence of a linear combination of the $Q_{0}, Q_{1}, Q_{2}$ to a square and the number of coefficients which are zero is independent of the $\mathbb{C}^{*}$ action on $l$, $\left.Q_{1}, Q_{2}\right)$ and the fact that under this action $\left(\mathbb{C}^{*}\right)^{3} \times M_{0}$ has values in some irreducible component of $M$, which has to be $M_{0}$.

Now $\operatorname{pr}(M) \backslash \operatorname{pr}(N)$ defines a quasi projective subvariety $V^{\prime} \subset \mathcal{C}(1,2,2)$ of codimension one. Our aim is to construct a quasi projective variety $V \subset$ $\mathcal{C}(1,2,2)$ of codimension one, which is contained in $V^{\prime}$ satisfying the further conditions of Corollary 8.5(2), and hence proving the Theorem. We already chose $M$ and $N$ in a way that $V^{\prime}$ satisfies condition (3) of 8.4. All of the configurations which had to be excluded because of the further conditions in 8.4 and 8.5 define a proper algebraic subset $W \subset \mathcal{C}(1,2,2)$. All we need is to see that $V:=V^{\prime} \backslash W$ is not empty. But we have $\operatorname{pr}\left(m_{0}\right) \in V^{\prime} \backslash W$ for our point $m_{0}$ coming from the example below.

Example 8.12 The following set of quadratic polynomials defines an element of $V$. In particular the complement of its zero-sets in $\mathbb{P}_{2}$ is complete hyperbolic and hyperbolically embedded.

$$
\begin{aligned}
& Q_{0}=z_{0}^{2} \\
& Q_{1}=z_{1}^{2}+z_{0} z_{1}+z_{0} z_{2}+(1 / 25) z_{1} z_{2} \\
& Q_{2}=z_{2}^{2}+50 z_{0} z_{1}-10 z_{0} z_{2}+9 z_{1} z_{2}
\end{aligned}
$$

One checks immediately that $225 Q_{0}+100 Q_{1}+4 Q_{2}$ is a square. Set $\Gamma_{j}=$ $V\left(Q_{j}\right)$. Furthermore:

1) No more than two $\Gamma_{j}$ intersect in one point.

2) None of the $\Gamma_{j}$ are tangent to any other $\Gamma_{k}$. 
3) No tangent to one of $\Gamma_{2}$ and $\Gamma_{3}$ at a point of intersection with any $\Gamma_{j}$ contains a further point of intersection of the $\Gamma_{j}$.

4) No tangent to one of $\Gamma_{2}$ and $\Gamma_{3}$ at a point of intersection with $\Gamma_{1}$ is tangent to the other smooth quadric.

5) There exists no smooth quadric $\Gamma$ with $\Gamma_{2} \cap \Gamma=\left\{p^{\prime}\right\}, \Gamma_{3} \cap \Gamma=\left\{p^{\prime \prime}\right\}$ and $\left\{p^{\prime}, p^{\prime \prime}\right\} \subset \Gamma_{1}$.

How to check 1) to 4) is obvious. If $T^{\prime}$ resp. $T^{\prime \prime}$ are the linear polynomials which give the tangents at $\Gamma_{2}$ in $p^{\prime}$ resp. at $\Gamma_{3}$ in $p^{\prime \prime}$ we have $Q=a Q_{1}+b\left(T^{\prime}\right)^{2}$, $Q=c Q_{3}+d\left(T^{\prime \prime}\right)^{2}$, where $\Gamma=V(Q), \Gamma_{i}=V\left(Q_{i}\right)$. Now solve for $a, b, c, d$, and show that only the trivial solution exists.

For intersections of three smooth quadrics Theorem 8.4 is not quite superseeded by the more general statement of Theorem 7.1. as the application to intersections to Fermat quadrics shows. We first note a further corollary to Theorem 8.4.

Corollary 8.13 Let $\Gamma_{j}=V\left(Q_{j}\right) \subset \mathbb{P}_{2}, j=1,2,3$ be smooth quadrics, and let the assumptions of 8.4 be satisfied.

(1) The quasiprojective variety $\mathbb{P}_{2} \backslash \bigcup_{j=1}^{3} \Gamma_{j}$ is Brody-hyperbolic, unless there exists a smooth quadric or a line $\Gamma$ such that after choosing the notation accordingly ( $p, q$ distinct points):

(a) $\Gamma \cap \Gamma_{1}=\{p, q\}, \Gamma \cap \Gamma_{2}=\{p\}, \Gamma \cap \Gamma_{3}=\{q\}$

(b) $\Gamma \cap \Gamma_{1}=\{p\}, \Gamma \cap \Gamma_{2}=\{p\}, \Gamma \cap \Gamma_{3}=\{q\}$

(2) The above conditions (a) and (b) can be replaced by the following (somewhat stronger) condition:

(c) all of the $\Gamma_{j}$ intersect transversally.

In this case $\mathbb{P}_{2} \backslash \bigcup_{j=1}^{3} \Gamma_{j}$ is complete hyperbolic and hyperbolically embedded.

We apply the Corollary to the following

Proposition 8.14 Let

$$
Q_{j}=a_{j} x^{2}+b_{j} y^{2}+c_{j} z^{2} ; \quad j=1,2,3
$$

be linearly independent polynomials, whose zero-sets $\Gamma_{j}$ are smooth. Assume 
(1) no more than two of the $\Gamma_{j}$ intersect at one point,

(2) no tangent to a quadric $\Gamma_{j}$ at a point of intersection with some other $\Gamma_{k}$ contains a further intersection point of the curves $\Gamma_{l}$,

(3) none of the $\Gamma_{j}$ are tangent to each other at any point.

Then $\mathbb{P}_{2} \backslash \cup_{j=1}^{3} \Gamma_{j}$ is complete hyperbolic and hyperbolically embedded.

\section{References}

[1] Adachi, Y., Suzuki, M., On the family of holomorphic mappings into projective space with lacunary hypersurfaces, J. Math. Kyoto Univ. 30 (1990), 451-458.

[2] Adachi, Y., Suzuki, M., Degeneracy Points of the Kobayashi pseudodistances on complex manifolds, Proc. Symp. Pure Math. 52 (1991), 41-51.

[3] Babets, V. A., Theorems of Picard type for holomorphic mappings, Siberian Math. J. 25 (1984), 195-200.

[4] Fulton, W., Algebraic curves, W.A.Benjamin, Inc. New York (1969).

[5] Grauert, H., Jetmetriken und hyperbolische Geometrie, Math. Z. 200 (1989), 149-168.

[6] Green, M., On functional equation $f^{2}=e^{2 \phi_{1}}+e^{2 \phi_{2}}+e^{2 \phi_{3}}$ and a new Picard theorem, Transact. AMS 195 (1974), 223-230.

[7] Green, M., Some Picard theorems for holomorphic maps to algebraic varieties, Amer. J. Math. 97 (1975), 43-75.

[8] Green, M., The hyperbolicity of the complement of $2 \mathrm{n}+1$ hyperplanes in general position in $\mathbb{P}_{n}$ and related results, Proc. Am. Math. Soc 66 (1977), $109-113$.

[9] Hayman, W.K., Meromorphic functions, Oxford University Press (1964).

[10] Kobayashi, S., Hyperbolic manifolds and holomorphic mappings, Marcel Dekker, New York (1970). 
[11] Lang, S., Hyperbolic and diophantine analysis, Bull. Am. Math. Soc. 14, (1986), 159-205.

[12] Lang, S., Introduction to complex hyperbolic spaces, Springer-Verlag, New York (1987).

[13] Shiffman, B., On holomorphic curves and meromorphic maps in projective space, Indiana University Math. J. 28, (1979), 627-641.

[14] Stoll, W., The Ahlfors-Weyl theory of meromorphic maps on parabolic manifolds, Value Distribution Theory, Proceedings Joensuu 1981 (Ed. J.Laine, S.Rickman), LNM 981.

[15] Wong, P.M., On the second main theorem of Nevanlinna theory, Am. J. Math. 111 (1989), 549-583.

[16] Zaidenberg, M., Stability of hyperbolic embeddedness and construction of examples, Math. USSR. Sbornik 63, (1989), 351-361. 
Gerd Dethloff

Mathematisches Institut der Universität Göttingen

Bunsenstraße 3-5

3400 Göttingen

Germany

e-mail: DETHLOFF@CFGAUSS.UNI-MATH.GWDG.DE

Georg Schumacher

Ruhr-Universität Bochum, Fakultät für Mathematik

Universitätsstraße 150

4630 Bochum 1

Germany

e-mail:GEORG.SCHUMACHER@RUBA.RZ.RUHR-UNI-BOCHUM.DE

Pit-Mann Wong

Dept. of Mathematics, University of Notre Dame

Notre Dame, Indiana 46556

USA

e-mail:PMWONG@CARTAN.MATH.ND.EDU 